\title{
Real Gas Simulation of Hydrogen Release from a High-Pressure Chamber
}

\author{
Kaveh Mohamed $^{1}$, Marius Paraschivoiu ${ }^{1 *}$ \\ ${ }^{1}$ Department of Mechanical and Industrial Engineering, Concordia University, 1455 de Maisonneuve Blvd. West, \\ Montreal, Quebec
}

"Corresponding author, paraschi@me.concordia.ca

\begin{abstract}
Hydrogen release from a high-pressure chamber is modeled in this paper. Two approaches are developed to investigate the real gas effects at high pressures. In the first method, an analytical model is developed to simulate time histories of stagnation properties of hydrogen inside the chamber as well as sonic properties of hydrogen at the orifice. Corresponding thermodynamic relations, which describe the specific heats, internal energy, and speed of sound, are derived based on the Beattie-Bridgeman state equation. In the second approach, a 3-D unstructured tetrahedral finite volume Euler solver is applied to numerically simulate the hydrogen release. The solver is modified to take into account the real gas effects. Modifications required to calculate the real gas Jacobian matrices and eigenvectors as well as to obtain the Roe's averaged convective fluxes are described. Real gas effect is modeled by the same state equation. Numerical and analytical results are compared for ideal and real gas conditions. An excellent agreement is reported.
\end{abstract}

\section{INTRODUCTION}

Hydrogen, as a fuel, has some attractive features in terms of the low amount of pollution and high combustion efficiency, especially when it is used in a fuel cell to generate electricity. These features make hydrogen a candidate energy currency to replace hydrocarbon fuels in vehicles.

The problem with using hydrogen as a fuel is the storage. Compared to natural gas, hydrogen has smaller energy content per mole, and less moles of hydrogen can be stored in a given volume at the same pressure. The compressibility factor for hydrogen increases to values well above unity (ideal value) at high pressures. This implies that hydrogen should be stored at higher pressures compared to natural gas in order to reserve a reasonable amount of fuel in the vehicle.

For safety issues it is important to determine how the gas is released in case of failure. The worst most probable failure is the separation of a fitting in a high-pressure tube or pipe, which would result in a sudden release of hydrogen. The release of hydrogen into the air yields a detonable cloud [10]. In such conditions, real gas laws should be applied to calculate the flow rate of hydrogen releasing into the air. 
In this paper, real gas effects at high pressures are investigated in the sample problem of the hydrogen release from a high-pressure chamber. Two different approaches are applied. The first one involves the development of a mathematical model to calculate time histories of the stagnation properties inside the chamber and sonic properties at the throat. Real gas behavior is modeled by the Beattie-Bridgeman equation of state. Johnson [2] applied the same method with a different equation of state to tabulate thermodynamic properties of nitrogen and helium at pressures up to $30 \mathrm{MPa}$. In the second approach, an in house 3-D finite volume code is modified to take into account the real gas behavior. Jacobians and eigenvector matrices are derived based on our reference equation of state. Roe's averaging scheme is modified according to the method developed by Glaister and Abgrall's [3, 4] for 1-D and 2-D Riemann solvers.

The reminder of this paper is as follows. The analytical model is developed in Section 2. Thermodynamic relations are derived to calculate specific heats, the speed of sound, internal energy, and the isentropic expansion in a real gas flow. The numerical approach is described in Section 3 where Jacobian matrices, eigenvectors and modified Roe's averaging method are presented. Section 4 is devoted to the discussion of the analytical and numerical simulation results.

\section{Analytical Model}

This section starts by stating the assumptions of the flow studied and continues by derivation of the equations required to calculate the gas release flow properties. The following assumptions have been made to simulate the gas release from a high-pressure chamber:

- Thermodynamic properties are distributed uniformly throughout the chamber;

- The hydrogen release is simulated for adiabatic conditions where no heat transfer occurs between the gas inside the chamber and its surrounding container;

- The orifice is at the critical condition (i.e., the velocity of the gas at the orifice is equal to the local speed of sound);

- The expansion of the hydrogen from the stagnation state inside the chamber to the critical state at the orifice takes place at a small region near the orifice, and it is modeled by a quasi one-dimensional isentropic flow;

- Hydrogen is assumed to exist in gaseous phase through the whole chamber;

- The real gas behavior is modeled by the Beattie-Bridgeman state equation. All required constants of the equation, $A_{\circ}, B_{\circ}, \alpha, b$, and $c$, are listed in Table 1.

$$
\begin{aligned}
P & =f(T, v) \\
& =\frac{R T}{v}+\left(\frac{-c R}{T^{2}}+B_{\circ} R T-A_{\circ}\right) \frac{1}{v^{2}}+\left(-\frac{B_{\circ} c R}{T^{2}}-B_{\circ} b R T+\alpha A_{\circ}\right) \frac{1}{v^{3}}+\frac{B_{\circ} b c R}{T^{2}} \frac{1}{v^{4}} .
\end{aligned}
$$

Pressure, temperature, and specific volume of the gas are denoted by $P, T$, and $v$. The gas constant is given by $R$.

Although the first and fourth assumptions seem contrary to each other, they will give reasonable results for a large high-pressure chamber with a small orifice, [1]. The third assumption is acceptable if the total time of the gas release is not a concern. After the flow reaches subsonic speeds at the orifice, pressure, density, and temperature-changes become very small and negligible compared to corresponding values that exist when the flow is sonic at the orifice. 
Table 1: Constants of the Beattie-Bridgeman equation of state for Hydrogen

\begin{tabular}{ccccc}
\hline$A_{\circ}$ & $10^{+3} \alpha$ & $10^{+2} B$ & $10^{+2} b$ & $10^{-2} c$ \\
\hline 4.924 & -2.510 & 1.034 & -2.162 & 2.500 \\
\hline
\end{tabular}

\subsection{Conservation Equations}

A mathematical model developed herein to calculate the release of hydrogen is constructed by applying conservations of mass and energy to the control volume containing the gas inside the chamber. The model describes the physical process that the bulk of the inside gas undergoes. The conservation of mass is given by:

$$
\frac{\partial}{\partial t}\left(\frac{\Psi}{v_{t}}\right)=-\frac{a_{n} A_{n}}{v_{n}} .
$$

In an adiabatic release, the conservation of energy can be written as:

$$
\frac{\partial}{\partial t}\left(\frac{\Psi i_{t}}{v_{t}}\right)=-\frac{h_{n} a_{n} A_{n}}{v_{n}} .
$$

Chamber volume and surface area of the throat are shown by $\Psi$ and $A_{n}$. Internal energy and enthalpy of the flow are denoted by $i$ and $h$. The physical time is given by $t$. Subscripts ${ }_{\mathrm{t}}$ and $\mathrm{n}_{\mathrm{n}}$ show that the corresponding property is evaluated at the stagnation state inside the chamber and the sonic state at the throat, respectively.

After expanding derivatives on the left hand sides, equations (2) and (3) can be written into the following forms:

$$
\begin{aligned}
\frac{\partial v_{t}}{\partial t} & =\frac{v_{t}^{2}}{\Psi} \frac{a_{n} A_{n}}{v_{n}}, \\
\frac{\partial i_{t}}{\partial t} & =-\frac{\mathbf{Q}_{n}+0.5 a_{n}^{2} a_{n} A_{n}}{\Psi} \frac{v_{t}}{v_{n}}+\frac{i_{t} a_{n} A_{n}}{\Psi} \frac{v_{t}}{v_{n}} .
\end{aligned}
$$

Thermodynamic properties of the flow crossing the orifice appear on the right hand sides of conservation equations (4) and (5). The method to calculate these properties is described in the following subsections. First, relations between dependent and independent thermodynamic properties are defined then an expression that governs the isentropic expansion or compression is developed.

\subsection{Some Real Gas Thermodynamic Relations}

In order to keep consistency with the state equation (1), temperature and specific volume are selected as independent thermodynamic properties. Speed of sound, specific heats, internal energy and enthalpy are calculated in terms of temperature and specific volume. Mathematically, different relations between dependent and independent properties can be obtained if another form of the state equation is utilized, [2].

For a pure substance, the following relations exist between changes in entropy, temperature, pressure, and specific volume [6]: 


$$
d s=C_{v} \frac{d T}{T}+\left(\frac{\partial P}{\partial T}\right)_{v} d v=C_{P} \frac{d T}{T}-\left(\frac{\partial v}{\partial T}\right)_{P} d P
$$

Entropy of the gas is expressed by $s . C_{v}$ and $C_{P}$ are constant-volume and constant- pressure specific heats, respectively. The subscripts of the partial derivatives, $v$ and $P$, show that the specific volume and pressure are kept constant, respectively, during the partial differentiation. In an isentropic process, where there is no change in entropy, equation (6) yields:

$$
\begin{aligned}
& \left(\frac{\partial T}{\partial v}\right)_{s}=-\frac{T}{C_{v}}\left(\frac{\partial P}{\partial T}\right)_{v}, \\
& \left(\frac{\partial T}{\partial P}\right)_{s}=\frac{T}{C_{P}}\left(\frac{\partial v}{\partial T}\right)_{P} .
\end{aligned}
$$

\subsubsection{Specific Heats}

Constant-pressure specific heat of hydrogen at a reference pressure of $0.1 \mathrm{MPa}$, which is denoted by $\tilde{C}_{P}$, is assumed to remain constant during the gas release for a range of temperature between $300^{\circ} \mathrm{K}$ and $60^{\circ} \mathrm{K}$. There is a maximum relative error of $\pm 4 \%$ between the assumed averaged value and actual values of specific heat tabulated in [5]. Since hydrogen behaves as an ideal gas at the reference pressure, the averaged specific heat must be corrected to obtain the corresponding value at high pressures.

Given constant-pressure specific heat of hydrogen, $\tilde{C}_{P}$, at temperature $T$ and the reference pressure of $0.1 \mathrm{MPa}$, we calculate constant-volume specific heat, $C_{v}$, at a final highpressure state, which is specified by temperature $T$ and specific volume $v$. Constant-volume specific heat and specific volume of hydrogen at the reference pressure of $0.1 \mathrm{MPa}$ and temperature $T$ can be calculated using ideal gas equation of state,

$$
\begin{aligned}
& v^{\circ}=\frac{R T}{100000}, \\
& \tilde{C}_{v}=\widetilde{C}_{P}(T)-R .
\end{aligned}
$$

Considering a constant-temperature process from the reference state to the final high-pressure state, we modify the constant-volume specific heat as:

$$
C_{v}=\tilde{C}_{v}+\int_{v^{\circ}}^{v}\left(\frac{\partial C_{v}}{\partial v}\right)_{T} d v
$$

According to [6], the following relation exists between specific heat and partial derivatives of pressure in a pure substance:

$\left(\frac{\partial C_{v}}{\partial v}\right)_{T}=T\left(\frac{\partial^{2} P}{\partial T^{2}}\right)_{v}$

Substituting equation (12) into equation (11), we have:

$$
C_{v}(T, v)=\tilde{C}_{v}+\int_{v^{\circ}}^{v} T\left(\frac{\partial^{2} P}{\partial T^{2}}\right)_{v} d v=\tilde{C}_{v}+\int_{v^{\circ}}^{v} T f_{T T}(T, v) d v
$$

In a pure substance, the relation between constant-volume and constant-pressure specific heats is given by, [6]: 


$$
C_{P}-C_{v}=-T\left(\frac{\partial v}{\partial T}\right)_{P}^{2}\left(\frac{\partial P}{\partial v}\right)_{T}=-T\left(\frac{\partial P}{\partial T}\right)_{v}^{2} /\left(\frac{\partial P}{\partial v}\right)_{T} .
$$

Equation (14) is applied to calculate constant-pressure specific heat as:

$$
C_{P}(T, v)=C_{v}(T, v)-T \frac{f_{T}^{2}(T, v)}{f_{v}(T, v)} \text {. }
$$

Subscripts $v$ and ${ }_{T}$ represent partial derivatives of the function $f \boldsymbol{\mathcal { C }}, v_{-}^{-}$, with respect to specific volume and temperature, respectively.

\subsubsection{Speed of Sound}

By definition, sound velocity, $a$, is given by:

$$
a=\sqrt{\left(\frac{\partial P}{\partial \rho}\right)_{s}}=\sqrt{-v^{2}\left(\frac{\partial P}{\partial v}\right)_{s}}=\sqrt{-v^{2} \frac{Q T / \partial v}{Q T / \partial P}} \text {. }
$$

Substituting isentropic derivatives (7) and (8) into equation (16) gives:

$$
a(T, v)=\sqrt{v^{2} \frac{C_{P}}{C_{v}} \frac{Q P / \partial T_{\psi}}{\partial v / \partial T_{D}}}=\sqrt{-v^{2} \frac{C_{P}}{C_{v}}\left(\frac{\partial P}{\partial v}\right)_{T}}=\sqrt{-v^{2} \frac{C_{P}}{C_{v}} f_{v}(T, v)} .
$$

\subsubsection{Internal Energy}

In [6], the variation of the internal energy of a pure substance is given by:

$$
d i=C_{v} d T+\left[T\left(\frac{\partial P}{\partial T}\right)_{v}-P\right] d v
$$

Internal energy and enthalpy of hydrogen as a real gas can be written as:

$$
\begin{aligned}
& i(T, v)=\tilde{C}_{v} T+\int_{v^{\circ}}^{v} \mathbf{f} f_{T}(T, v)-f(T, v) \underline{d} v, \\
& h(T, v)=i(T, v)+v f(T, v) .
\end{aligned}
$$

Thermodynamic relations developed in this section are in terms of derivatives and integrals of state equation (1). Table 2 contains analytical expressions of these derivatives and integrals.

\subsubsection{Isentropic Expansion}

Hydrogen goes through an isentropic expansion from the stagnation state in the chamber to the sonic state at the orifice. Properties of the isentropically expanded flow can be determined by integrating equation (7) with respect to the specific volume.

$d T=-\frac{T f_{T}(T, v)}{C_{v}} d v$.

Starting from the initial condition that corresponds to the stagnation state inside the chamber, we integrate equation (21) to find thermodynamic states the gas goes through during an isentropic expansion. The sonic state of the flow at the throat is the state at which the following balance of energy is satisfied:

$$
i\left(T_{t}, v_{t}\right)+v_{t} f\left(T_{t}, v_{t}\right)=i\left(T_{n}, v_{n}\right)+v_{n} f\left(T_{n}, v_{n}\right)+\frac{1}{2} a^{2}\left(T_{n}, v_{n}\right) .
$$


The last two equations are used to uniquely determine sonic properties of hydrogen at the orifice.

\subsection{Computer Code}

A computer program is developed to calculate stagnation and sonic properties of hydrogen during the release from a high-pressure chamber. It is based on relations developed in Section 2. The program comprises two major steps: isentropic expansion and adiabatic release. Fig. 1 shows the flowchart of the program's algorithm. $\mathrm{P}_{\text {back }}$ is the atmospheric backpressure to which hydrogen discharges.

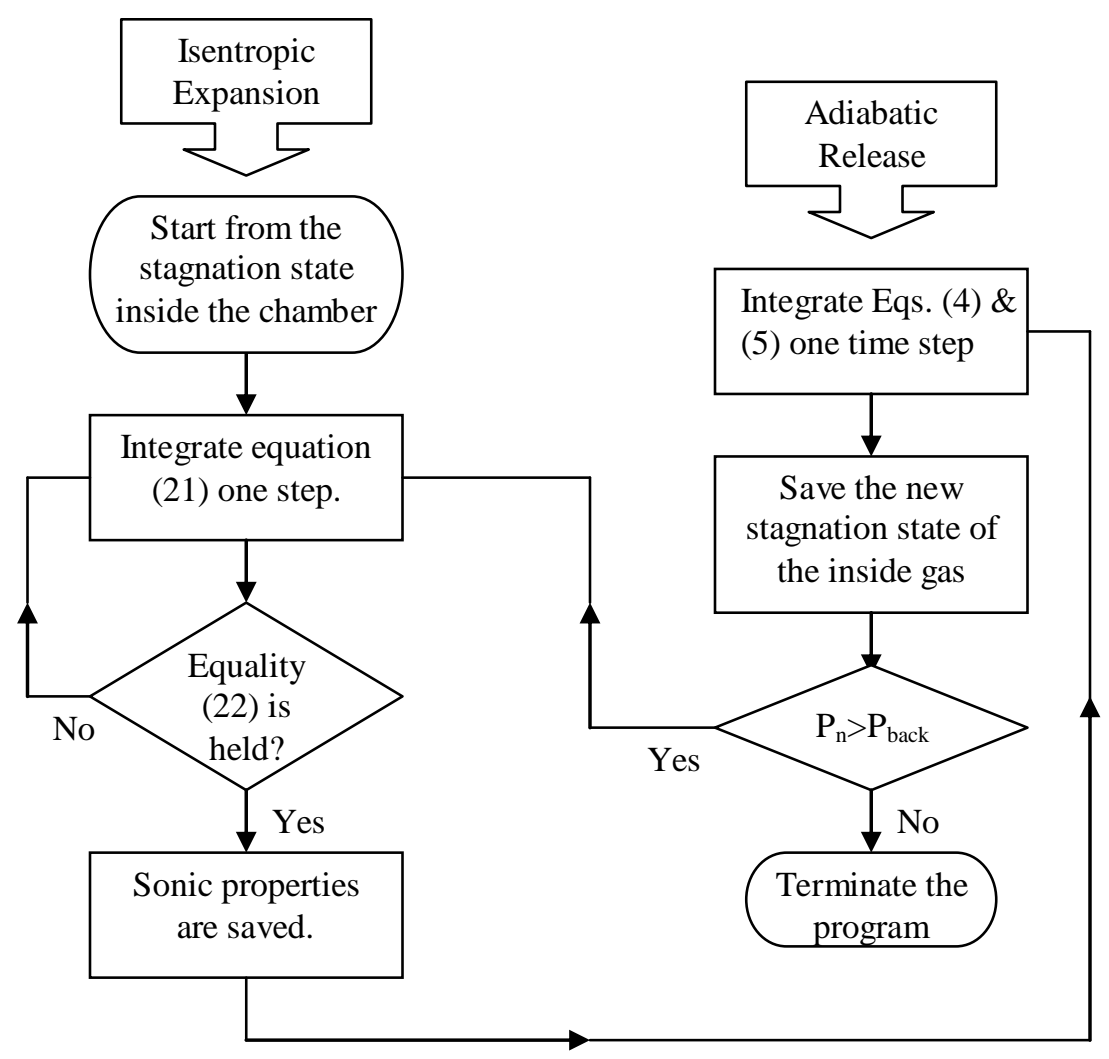

Figure 1: Flowchart of the adiabatic gas release algorithm

\subsubsection{Isentropic Expansion}

This step involves the numerical integration of ordinary differential equation (21). The domain of integration consists of all expanded states hydrogen goes through, from the stagnation state inside the chamber to the sonic state at the orifice. The initial condition is determined from the current stagnation state inside the chamber, and the final condition is reached once equality (22) is satisfied. After each integration step, equation (22) is checked. Its right hand side is evaluated at the most recent computed values of specific volume and temperature.

A first order Euler method is applied to integrate the ordinary differential equation (21). 


\subsubsection{Adiabatic Release}

In an adiabatic gas release, the system of ordinary differential equations (4) and (5) are numerically solved using the first order Euler method. The initial condition is the initial stagnation state inside the chamber. Numerical integration stops when the pressure at the orifice reaches to the backpressure. The velocity of the flow at the orifice always remains sonic. Each integration step results in new values of specific volume and internal energy. The corresponding new value of temperature is obtained through an iterative solution of equation (19). Iterations are performed based on the secant method.

\section{NUMERICAL APPROACH}

A 3-D finite volume Euler solver, [7], is used to simulate the gas release from a 60-degree wedge of a high-pressure chamber. The unstructured tetrahedral mesh is generated using GAMBIT 2.0.4, fig 2. It contains 2452 mesh points and 9712 tetrahedral elements. The viscosity is neglected and Euler equations are applied through an implicit first-order in time and secondorder in space scheme. Convective fluxes are calculated based on Roe's averaging method, [8]. As all Jacobian matrices and Roe's averaging method that were already applied to the code are in terms of ideal gas relations, modifications described in the following subsections have been done to model the real gas effects. Two types of boundary conditions are implemented. A free-slip boundary condition is applied to the walls and the cutting surfaces of the 60 -degree wedge. The exit surface is described by a supersonic outlet boundary condition.
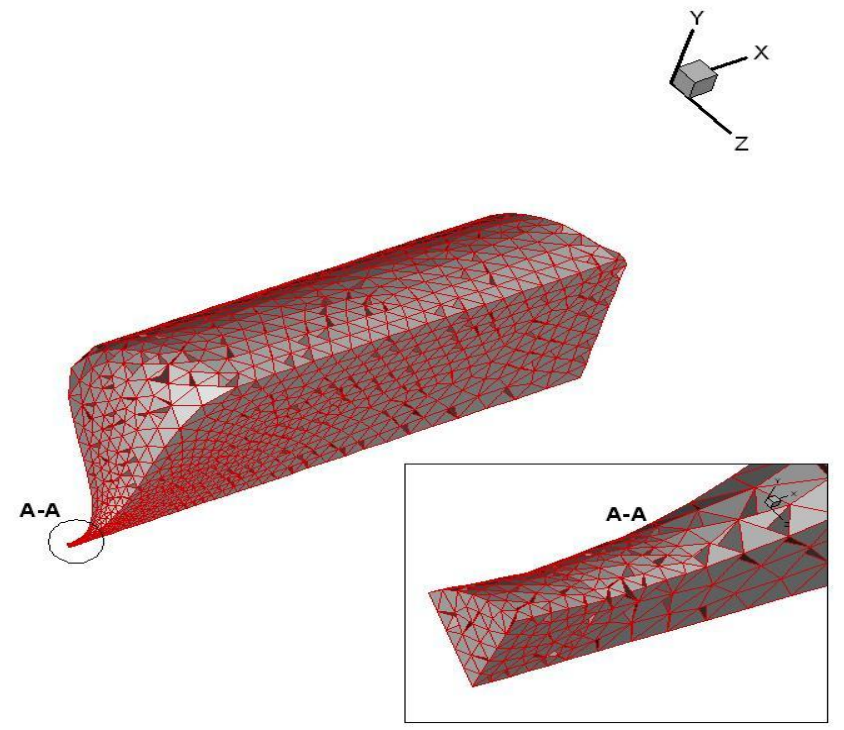

Figure 2: The 60-degree Wedge of the cylindrical chamber with unstructured tetrahedral mesh

The state equation is assumed to be implicitly a function of density, $\rho$, and internal energy, $i$, $P=P(\rho, i)$.

This assumption let us directly apply the method developed in $[3,4]$ to find Roe's averaging values. The partial derivative of pressure with respect to internal energy for constant density and the partial derivative of pressure with respect to density for constant internal energy are 
respectively represented by $P_{i}$ and $P_{\rho}$. These values can be equivalently expressed in terms of the derivatives and integrals of the Beattie-Bridgeman equation of state, [9]:

$$
\begin{aligned}
& P_{i}=\frac{f_{T}}{i_{T}}, \\
& P_{\rho}=v^{2}\left(\frac{f_{T} i_{v}}{i_{T}}-f_{v}\right),
\end{aligned}
$$

where $i_{T}$ and $i_{v}$ are partial derivatives of equation (19) with respect to temperature and specific volume, respectively. Analytical expressions are given in Table 2.

\subsection{Conservation Equations}

The system of Euler equations is discretized using an implicit finite-volume discretization scheme,

$$
\left\|\tau_{j}^{c}\right\| \frac{\vec{U}_{j}^{n+1}-\vec{U}_{j}^{n}}{\Delta t}+\sum_{\text {over } \delta \tau_{j}^{c}} \overrightarrow{\vec{F}}_{\delta \tau_{j}^{c}}^{n+1} \cdot \vec{n}_{\delta \tau_{j}^{c}} \Delta S_{\delta \tau_{j}^{c}}=0
$$

where $j$ goes from 1 to the number of control volumes (nodes) that exist in the domain of computation. $\vec{U}$ is the vector of conservative variables. Conservative fluxes crossing the boundary surfaces are represented by $\overrightarrow{\vec{F}} . \vec{n}$ is the unit vector normal to the boundary surface with an area of $\Delta S$. The calculation time step is denoted by $\Delta t$.

Using the first order approximation for implicit fluxes, we can rewrite equation (26) in the following forms:

$$
\begin{aligned}
& \left\{\frac{\left\|\tau_{j}^{c}\right\|}{\Delta t} \overrightarrow{\vec{I}}+\sum_{o v e r \delta \tau_{i}^{c}}\left(\frac{\partial \overrightarrow{\vec{F}}}{\partial \vec{U}}\right)_{\delta \tau_{i}^{c}}^{n} \cdot \vec{n}_{\delta \tau_{i}^{c}} \Delta S_{\delta \tau_{i}^{c}}\right\} \delta \vec{U}_{j}^{n+1}=-\sum_{o v e r \delta \tau_{i}^{c}} \overrightarrow{\vec{F}}_{\delta \tau_{i}^{c}}^{n} \cdot \vec{n}_{\delta \tau_{i}^{c}} \Delta S_{\delta \tau_{i}^{c}}, \\
& \vec{U}_{j}^{n+1}=\vec{U}_{j}^{n}+\delta \vec{U}_{j}^{n+1} .
\end{aligned}
$$

$\left(\frac{\partial \overrightarrow{\vec{F}}}{\partial \vec{U}}\right) \cdot \vec{n}$ is the flux Jacobian matrix in the direction of the normal vector $\vec{n}$. This matrix is represented by $\mathbf{I}_{-}^{-}$in the rest of this paper.

An iterative algorithm, GMRES method described in [11], is applied to solve equation (27) for $\delta \vec{U}_{j}^{n+1}$. The iteration stops when the residual is less than $10^{-6}$. After stopping the iterations, the values of the conservative variables at the new time step are calculated from equation (28).

\subsection{Jacobian Matrix}

The flux Jacobian of primitive variables and its eigenvectors do not depend on a specific state equation, [9]. They are used as a reference to calculate flux Jacobian and eigenvectors of conservative variables where the implicit state equation (23) describes the real gas behavior. 


\subsubsection{Conservative to primitive Jacobian}

Conservative and primitive variable vectors are given by:

$\vec{U}^{T}=$ Џ $\quad \rho u \quad \rho v \quad \rho w \quad \rho E_{,}^{-}$

$\vec{Q}^{T}=\mathbf{W} \quad u \quad v \quad w \quad P$,

where $u, v$, and $w$ are $\mathrm{x}, \mathrm{y}$, and z-components of the velocity. $E$ is the total energy of the flow, which is the sum of the internal energy and the kinetic energy. The superscript ${ }^{T}$ stands for the transpose of the corresponding vector or matrix. Conservative to primitive transformation matrix is denoted by matrix ${ }_{-}^{-}$, and given by, [9]:

$$
\boldsymbol{M} \exists\left[\frac{\partial \vec{U}}{\partial \vec{Q}}\right]=\left[\begin{array}{ccccc}
1 & 0 & 0 & 0 & 0 \\
u & \rho & 0 & 0 & 0 \\
v & 0 & \rho & 0 & 0 \\
w & 0 & 0 & \rho & 0 \\
i+\frac{p}{\rho}+\frac{V^{2}}{2}-\frac{\rho a^{2}}{p_{i}} & \rho u & \rho v & \rho w & \frac{\rho}{p_{i}}
\end{array}\right] .
$$

$V$ is the magnitude of the velocity vector. The inverse of the above matrix denoted by $\boldsymbol{M}^{-1}{ }^{-}$is the primitive to conservative transformation matrix,

$$
\boldsymbol{I}^{-1} \exists\left[\frac{\partial \vec{Q}}{\partial \vec{U}}\right]=\left[\begin{array}{ccccc}
1 & 0 & 0 & 0 & 0 \\
\frac{-u}{\rho} & \frac{1}{\rho} & 0 & 0 & 0 \\
\frac{-v}{\rho} & 0 & \frac{1}{\rho} & 0 & 0 \\
\frac{-w}{\rho} & 0 & 0 & \frac{1}{\rho} & 0 \\
a^{2}+\frac{P_{i}}{\rho} \mathbf{\gamma}^{2}-H & \frac{-u P_{i}}{\rho} & \frac{-v P_{i}}{\rho} & \frac{-w P_{i}}{\rho} & \frac{P_{i}}{\rho}
\end{array}\right] \text {, }
$$

where $H$ is the total enthalpy of the flow.

\subsubsection{Eigenvectors}

Left eigenvectors of primitive flux Jacobian matrix are given in [8]. They are represented here by symbols $\overrightarrow{\vec{l}}_{j}$ where $j$ goes from 1 to 5 . The left eigenvectors of conservative flux Jacobian are represented by $\vec{l}_{j}$ and can be calculated as:

$$
\vec{l}_{j}=\overrightarrow{\vec{l}}_{j} \mathbf{M}^{-1} \dot{-}
$$

The left eigenvector matrix $\mathbf{L}_{-}^{-}$is constructed so that its rows are the left eigenvectors given by $\vec{l}_{j}$, 


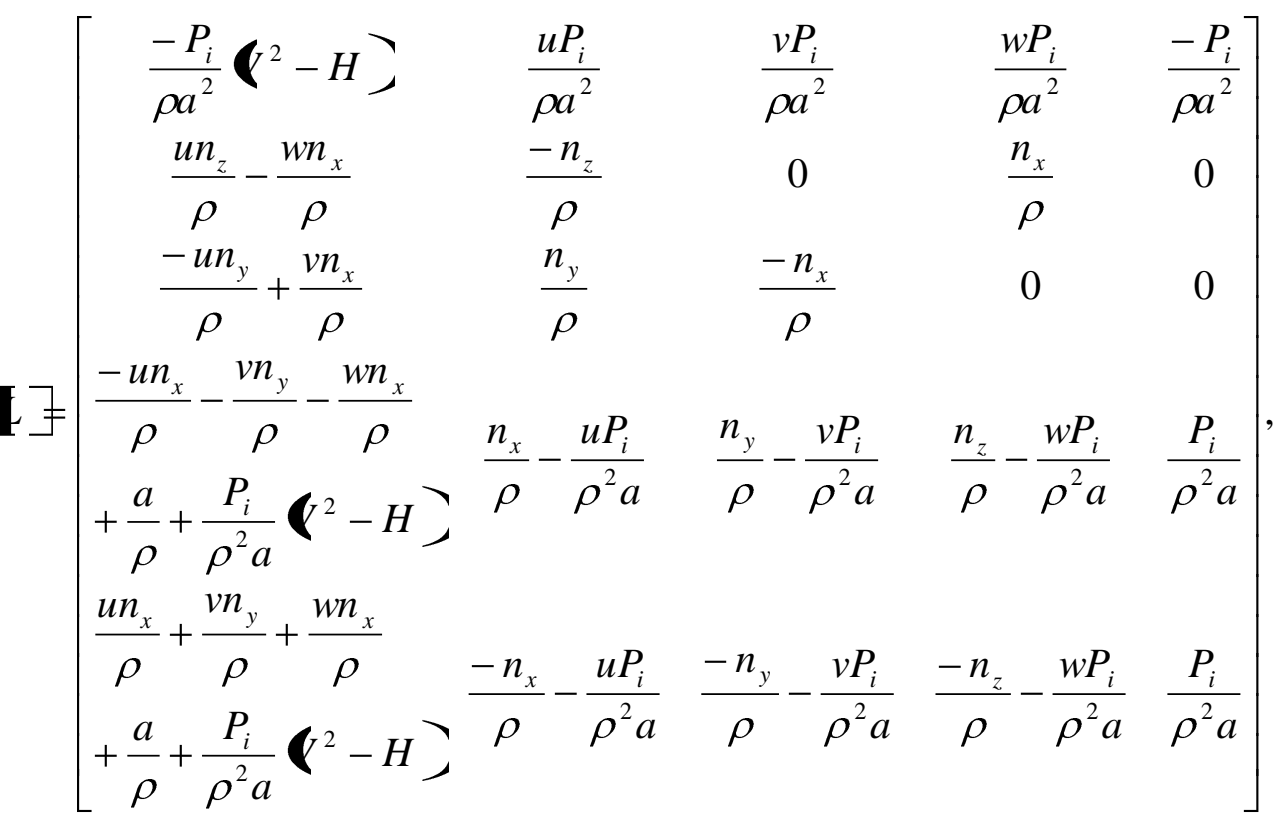

where $n_{x}, n_{y}$, and $n_{z}$ are $\mathrm{x}, \mathrm{y}$, and $\mathrm{z}$-components of the unit vector normal to the boundary surface between two nodes. The relation between conservative and primitive right eigenvectors, $\vec{r}_{j}$ and $\overrightarrow{\vec{r}}_{j}$, are given by:

$\vec{r}_{j}=\Lambda \overrightarrow{\vec{r}}_{j}$.

The corresponding matrix $\mathbf{R}_{-}^{-}$is a matrix whose columns are right eigenvectors of conservative flux Jacobian matrix,

$$
\mathbf{R}=\left[\begin{array}{ccccc}
1 & 0 & 0 & \frac{\rho}{2 a} & \frac{\rho}{2 a} \\
u & -\rho n_{z} & \rho n_{y} & \frac{\rho u}{2 a}+\frac{\rho n_{x}}{2} & \frac{\rho u}{2 a}-\frac{\rho n_{x}}{2} \\
v & -\frac{\rho n_{y} n_{z}}{n_{x}} & -\frac{\rho \mathbf{Q}_{z}^{2}+n_{x}^{2}-}{n_{x}} & \frac{\rho v}{2 a}+\frac{\rho n_{y}}{2} & \frac{\rho v}{2 a}-\frac{\rho n_{y}}{2} \\
w & \frac{\rho \mathbf{6}_{y}^{2}+n_{x}^{2}}{n_{x}} & \frac{\rho n_{y} n_{z}}{n_{x}} & \frac{\rho w}{2 a}+\frac{\rho n_{z}}{2} & \frac{\rho w}{2 a}-\frac{\rho n_{z}}{2} \\
H-\frac{\rho a^{2}}{p_{i}} & -\rho u n_{z}-\frac{\rho v n_{y} n_{z}}{n_{x}} & \rho u n_{y}-\frac{\rho v \mathbf{Q}_{z}^{2}+n_{x}^{2}}{n_{x}} & \frac{\rho H}{2 a}+\frac{\rho u n_{x}}{2}+\frac{\rho H}{2 a}-\frac{\rho u n_{x}}{2}- \\
& +\frac{\rho w \mathbf{l}_{y}^{2}+n_{x}^{2}}{n_{x}} & +\frac{\rho w n_{y} n_{z}}{n_{x}} & \frac{\rho v n_{y}}{2}+\frac{\rho w n_{z}}{2} & \frac{\rho v n_{y}}{2}-\frac{\rho w n_{z}}{2}
\end{array}\right] .
$$

The flux Jacobian of conservative variables, $\mathbf{I}_{-}^{-}$, is calculated as:

where $\mathbf{\Lambda}_{-}^{-}$is the diagonal eigenvalue matrix as defined in [8]. 


\subsection{Roe's Averaging}

Roe's average values are calculated according to the method developed in [3, 4], which introduces definitions of average pressure derivatives $\tilde{P}_{i}$ and $\tilde{P}_{\rho}$. All average values are specified by a tilde mark. Roe's conservative flux vector can be written as:

$\left.\overrightarrow{\widetilde{F}}=\frac{1}{2}\left(\vec{F}_{R}+\vec{F}_{L}\right)-\frac{1}{2}\right] \mathbf{W} \mathbf{\Xi}_{R}-\vec{U}_{L}$ 广

The vector $\vec{F}$ is the conservative flux vector as defined in [8]. Subscripts ${ }_{\mathrm{L}}$ or ${ }_{\mathrm{R}}$ show that the corresponding value is evaluated at the left or right hand side node, respectively.

Table 2: Analytical expressions of integrals and derivatives of state equation

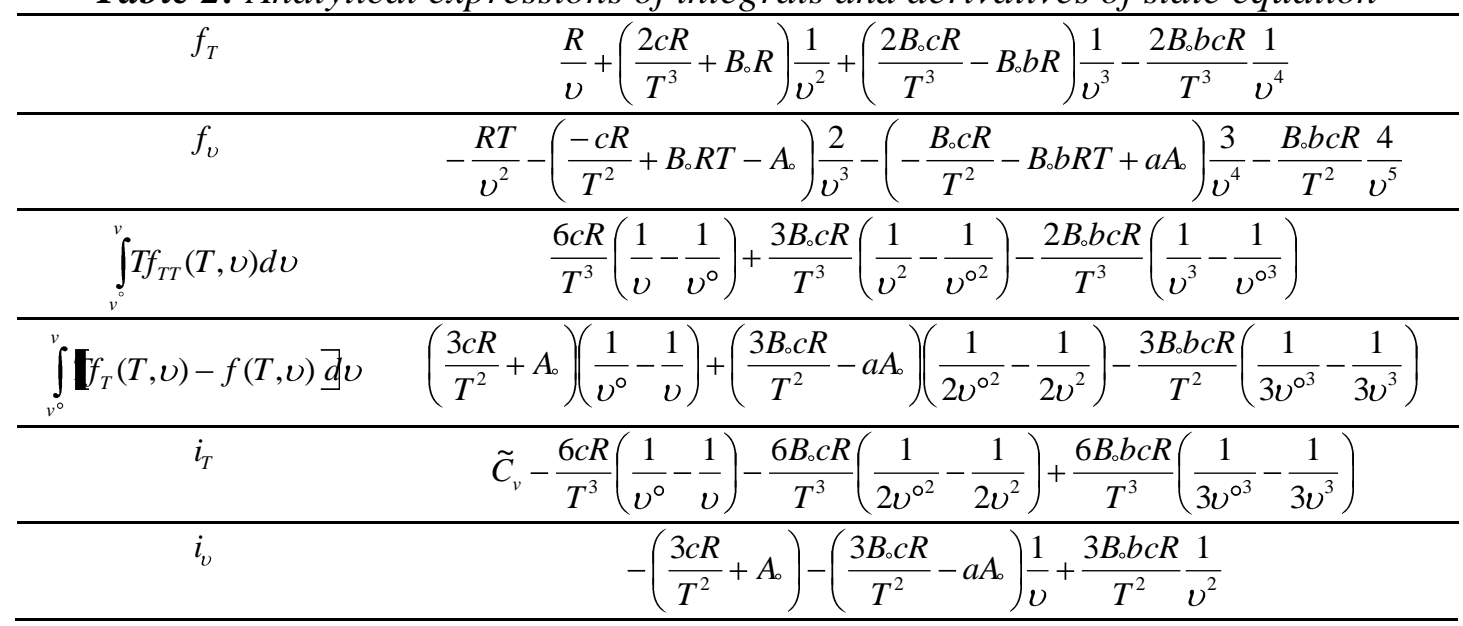

\subsection{Boundary Conditions}

The free slip boundary condition is applied to solid walls and cutting surfaces. Fluxes and Jacobians are evaluated directly at the boundary nodes without using Roe's averaging method. The flux vector and Jacobian at the free slip boundary are given in [9].

The exit surface is modeled by the supersonic-outlet boundary condition. If the conservative variables at the supersonic boundary are obtained from the extrapolation of interior nodes, CFL number should be kept less than 80 in order to avoid numerical instabilities. Whereas, no numerical instability is observed at CFL numbers up to 4000 when boundary points are solved together with the interior points using the system of equations (27) and (28). Roe's averaging method is dismissed at the boundary nodes, and fluxes and Jacobians are evaluated based on the boundary values.

\section{Results}

Some selected results of the analytical and numerical simulations are discussed in this section. Hydrogen releases from a high-pressure chamber with a volume of $2.73 \times 10^{-2} \mathrm{~m}^{3}$ and at initial pressure and temperature of $34.5 \mathrm{MPa}$ and $300{ }^{\circ} \mathrm{K}$. The gas adiabatically exits the chamber through a throat and an exit surface with surface areas of $3.17 \times 10^{-5} \mathrm{~m}^{2}$ and $3.73 \times 10^{-5} \mathrm{~m}^{2}$, 
respectively. The initial five seconds of the release are compared for ideal and real gas conditions In this laps of time, the total pressure drops from 34.5 MPa to less than 0.4 MPa while the throat always remains at the sonic condition with a back pressure of $0.1 \mathrm{MPa}$ and $98.5 \%$ of the initial mass inside the chamber is released. For the analytical model, equations (4) and (5) are integrated with a time-step of $10^{-6}$ sec. Numerical simulation starts with a CFL number of 1 that remains constant for the first 200 time steps. Then, it is incremented at a constant rate of ten units per time step to a final CFL value of 1000. The final value is kept unchanged until the end of the simulation.

The velocity, pressure, and temperature of the gas at the throat are depicted in figs 3-5. The numerical and analytical results for the real and ideal gas conditions are compared together. For both the real and the ideal gas simulation, a maximum relative error of $2 \%$ exists between the analytical and the numerical simulations.

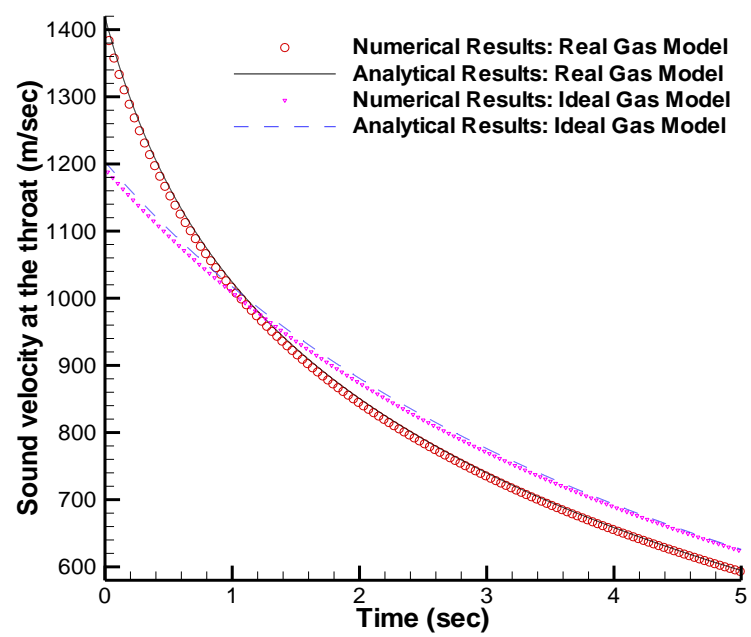

Figure 3: Analytical and numerical simulations of the flow velocity at the throat as a function of time for real and ideal gas conditions; hydrogen is released from a reservoir at initial pressure and temperature of $34.5 \mathrm{MPa}$ and $300{ }^{\circ} \mathrm{K}$.

Real gas model predicts higher values of the sound velocity at the start of the release compared to the ideal gas model, fig. 3. The internal energy of a real gas includes not only the ideal part, which is related to the translational kinetic energies of molecules, but also contributions of vibrational and rotational energies. As the gas isentropically expands from stagnation state inside the chamber to the sonic state at the orifice the pressure decreases and hydrogen approaches ideal gas behavior. The vibrational and rotational internal energies are transformed into the kinetic energy of the flow. At the start of the release, when the ideal and real gases expand from more and less the same stagnation state, the throat velocity for real gas condition is higher than the corresponding value for ideal gas condition. The sonic pressure decreases to lower values at the throat for the real gas condition, fig. 4, because the gas flow at the throat accelerates to higher velocities. 


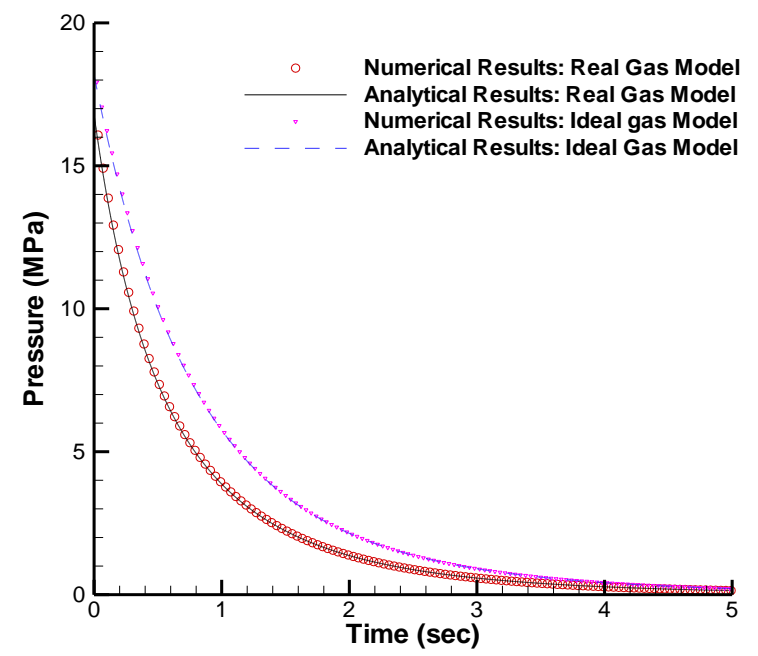

Figure 4: Analytical and numerical simulations of the flow pressure at the throat as a function of time for real and ideal gas conditions; hydrogen is released from a reservoir at initial pressure and temperature of $34.5 \mathrm{MPa}$ and $300 \%$.

A lower critical pressure at the throat for the real gas condition accompanies a lower critical temperature when compared to the ideal gas simulations for the throat temperature, fig. 5 .

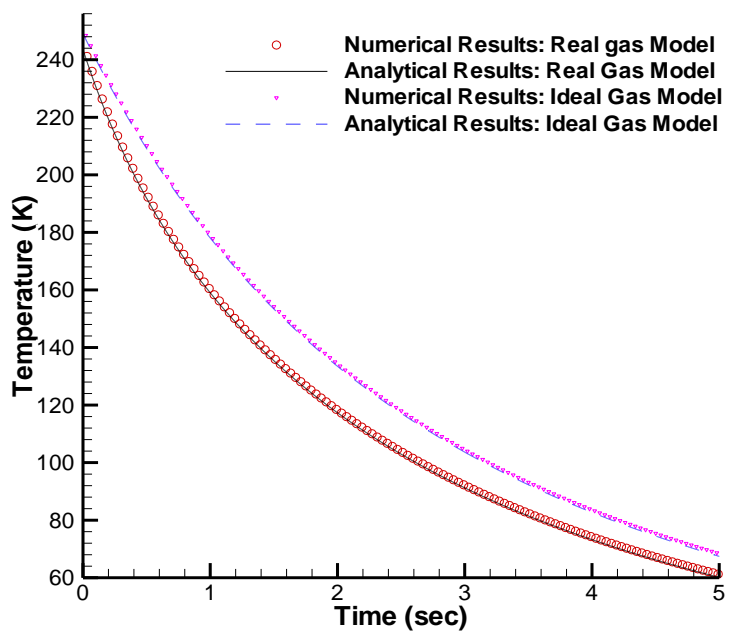

Figure 5: Analytical and numerical simulations of the flow temperature at the throat as a function of time for real and ideal gas conditions; hydrogen is released from a reservoir at initial pressure and temperature of $34.5 \mathrm{MPa}$ and $300{ }^{\circ} \mathrm{K}$.

The stagnation pressure and temperature of the gas inside the chamber are shown in figs. 6-7. Similar to the sonic properties, comparisons have been done between analytical and numerical predictions for real and ideal gas conditions. A maximum error of $1 \%$ exists between the analytical and the numerical simulations for both the real and the ideal gas models. 


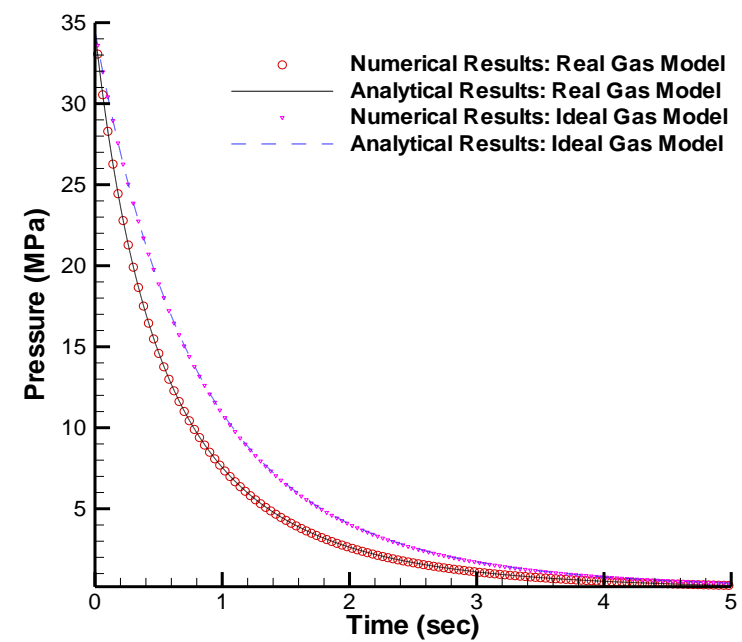

Figure 6: Analytical and numerical simulations of stagnation pressure inside the chamber as a function of time for real and ideal gas conditions; hydrogen is released from a reservoir at initial pressure and temperature of $34.5 \mathrm{MPa}$ and $300{ }^{\circ} \mathrm{K}$.

For real gas model, pressure, temperature and density decay more rapidly than those for ideal gas model. The initial higher flow velocity at the throat, results in higher amounts of mass and energy out-fluxes for the real gas model, which in turn lead to rapid decays of the stagnation pressure and temperature.

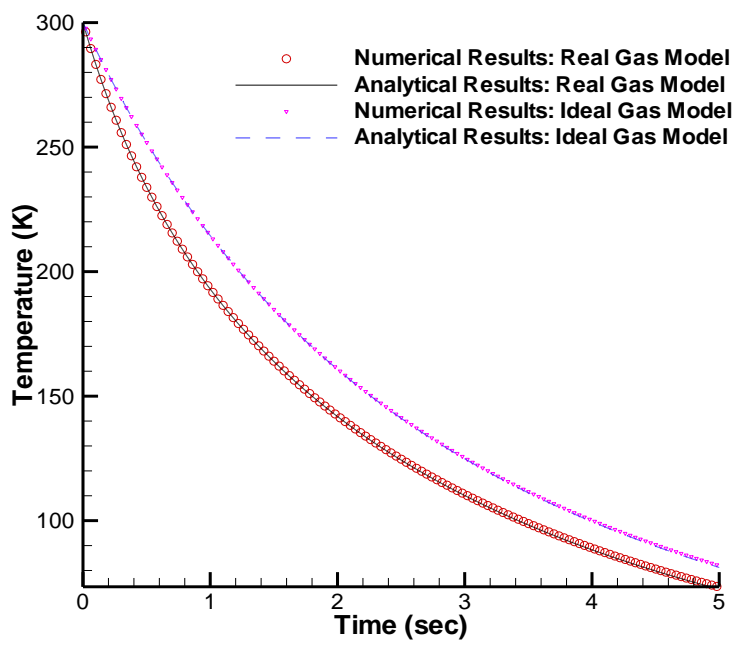

Figure 7: Analytical and numerical simulations of stagnation temperature inside the chamber as a function of time for real and ideal gas conditions; hydrogen is released from a reservoir at initial pressure and temperature of $34.5 \mathrm{MPa}$ and $300{ }^{\circ} \mathrm{K}$.

A colder and less pressurized stagnant gas isentropically expands to lower sonic velocities at the throat. As shown in fig. 3, the flow velocity at the throat for real gas model is 
higher than the corresponding values for ideal gas model during the initial moments of the release when both ideal and real gases are expanding from approximately the same stagnation pressures and temperatures, figs. 6-7. After these initial moments, when the real gas is expanding from significantly lower stagnation pressures and temperatures, the throat velocity predicted by the real gas model decreases and becomes less than the values predicted by the ideal gas model. The foregoing trend of the sonic velocity affects the relative behavior of real and ideal gas simulations for time histories of stagnation pressure or temperature, figs. 6-7. The curves of the real and ideal gas models are diverging from each other at the beginning of the release. The divergence rate decreases and curves start converging when the sonic velocity for real gas condition becomes less than the corresponding value for ideal gas condition.

The time history of the mass flow rate exiting the chamber is shown in fig. 8 . The density predicted by the ideal gas law is larger than the density the real gas law predicts. Therefore, for the ideal gas condition, the chamber initially contains more mass of hydrogen than that for real gas condition. Although the sonic velocity for real gas condition is higher than the corresponding value for the ideal gas condition, the high-density hydrogen flow predicted by the ideal gas model results in a greater mass flow rate throughout the release process.

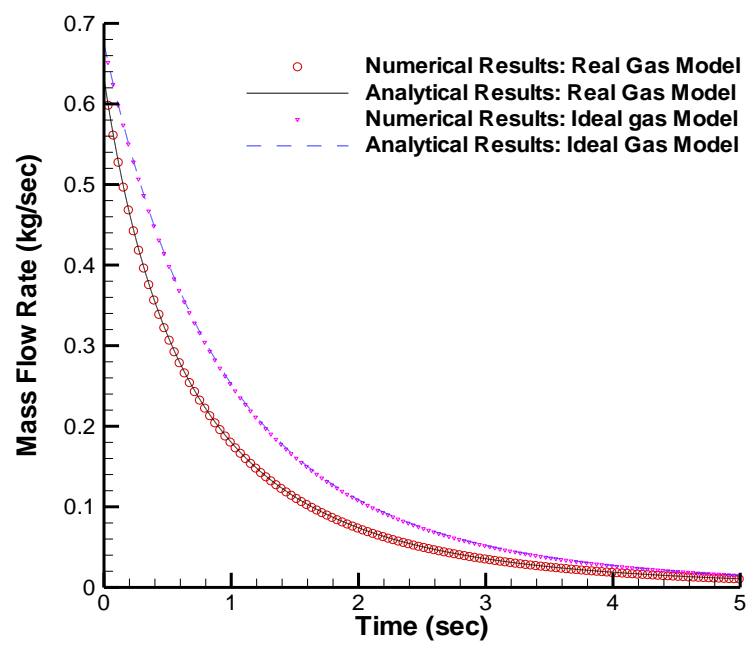

Figure 8: Analytical and numerical simulations of the mass flow rate exiting the chamber for real and ideal gas models as a function of time; hydrogen is released from a reservoir at initial pressure and temperature of $34.5 \mathrm{MPa}$ and $300{ }^{\circ} \mathrm{K}$.

\section{Conclusions}

Real gas effects are investigated in the adiabatic release of hydrogen from a high-pressure chamber. The analytical and numerical simulations are in a good agreement with each other. The maximum relative error is less than $2 \%$. The real gas behavior at high pressures results in a $14 \%$ increase in the velocity of the flow crossing the orifice at the start of the release. Stagnation and sonic pressure and temperature remain less than the corresponding ideal values throughout the release. The reduced stagnation pressure and temperature slow down the initially higher sonic velocity to lower values compared to the ideal gas. 


\section{REFERENCES}

[1] Saad M. Compressible Fluid Flow, $2^{\text {nd }}$ ed. Englewood Cliffs, New Jersey: Prentice-Hall, 1985.

[2] Johnson, R. C., Real-Gas Effects in Critical Flow through Nozzles and Thermodynamic Properties of Nitrogen and Helium at Pressures to 300 bars, Lewis Research Center, Cleveland, Ohio, NASA SP-3046, 1968.

[3] Glaister, P., An Approximate Linearized Riemann Solver for the Euler Equations for Real Gases, Journal of Computational Physics 74: 382-408, 1988.

[4] Abgrall, R., Extension of Roe's Upwind Scheme to Algebraic Equilibrium Real Gas Models, Computers and Fluids 19(2): 171-182, 1991.

[5] Journal of Physical and Chemical Reference Data: NIST-JANNAF Thermochemical Tables, $4^{\text {th }}$ ed., New York, New York: American Chemical Society and American Institute of Physics, 1998.

[6] Van Wylen, G. J., Sonntag, R. E., Fundamental of Classical Thermodynamics $2^{\text {nd }}$ ed., New York: John Wiley \& Sons, 1976.

[7] Xu, T., A Study of an ILU Preconditioned Multi-model Formulation for Compressible Flows, M.A.Sc. thesis, University of Toronto, Toronto, 2002.

[8] Hirsch, C., Numerical Computation of Internal and External Flows, Vol 2, New York: John Wiley \& Sons, 1992.

[9] Mohamed, K., Hydrogen Release from a High-Pressure Chamber Considering Real Gas Effects, M.A.Sc. Thesis, Concordia University, Montreal, 2004.

[10] Schmidt, D., Krause, U., Schmidtchen, U., Numerical Simulation of Hydrogen Gas Release Between Buildings, International Journal Hydrogen Energy 24: 479-488, 1999.

[11] Saad, Y., Schultz M. H., A Generalized Minimal Residual Algorithm for Solving Nonsymmetric Linear Systems, SIAM Journal of Scientific Computation 7: 856-869, 1986. 